\title{
Surgery Alone, Adjuvant Tegafur/gimeracil/octeracil (S-1), or Platinum-based Chemotherapies For Resectable Gastric Cancer: Real-world Experience and A Propensity Score Matching Analysis
}

\section{Chih-Chieh Yen}

Division of Hematology/ Oncology, Department of Internal Medicine, National Cheng Kung University Hospital Douliou Branch

\section{Yan-Shen Shan}

Department of Surgery, National Cheng Kung University Hospital, College of Medicine, National Cheng Kung University

\section{Yin-Jui Chao}

Department of Surgery, National Cheng Kung University Hospital, College of Medicine, National Cheng Kung University

\section{Ting-Kai Liao}

Department of Surgery, National Cheng Kung University Hospital, College of Medicine, National Cheng Kung University

\section{Hsuan-Yi Huang}

Division of Colorectal Surgery, Department of Surgery, Chi-Mei Medical Center

\section{I-Ting Liu}

Department of Oncology, National Cheng Kung University Hospital, College of Medicine, National Cheng Kung University

\section{Chia-Jui Yen ( $\nabla$ yencj@mail.ncku.edu.tw )}

Department of Oncology, National Cheng Kung University Hospital, College of Medicine, National Cheng Kung University

\section{Research Article}

Keywords: Adjuvant, Resectable Gastric Cancer, S-1, Platinum, Observation

Posted Date: February 10th, 2021

DOI: https://doi.org/10.21203/rs.3.rs-182845/v1

License: (c) (1) This work is licensed under a Creative Commons Attribution 4.0 International License. Read Full License 
Version of Record: A version of this preprint was published at BMC Cancer on July 9th, 2021. See the published version at https://doi.org/10.1186/s12885-021-08487-z. 


\section{Abstract}

Background: Adjuvant chemotherapy has changed the paradigm in resectable gastric cancer. S-1 is an oral chemotherapeutic with promising efficacy in Asia. However, comparisons with close observation or platinumbased doublets post $\mathrm{D} 2$ gastrectomy have been less reported.

Methods: We retrospectively evaluated patients with D2-dissected stage IB-III gastric cancer who received S-1 $(S-1, n=64)$, platinum-based doublets $(P, n=95)$ and operation with close observation (OBS, $n=110)$ from Jan 2013 to Oct 2018. A propensity score matching was used to compare for recurrence-free (RFS) and overall survivals (OS). Adverse reactions, dosage, and associated factors for S-1 are also discussed.

Results: In a median follow-up of 42.5 months, Group S-1 had a comparable survival to that of $\mathrm{P}$ and OBS. (median RFS/OS: S-1 vs. P, 24.4/37.4 vs. 39.1/62.1 months, HR=1.12/1.22, $p=0.667 / 0.514$; S-1 vs. OBS, $23.6 / 37.4$ vs. $24.0 / 64.6$ months, $\mathrm{HR}=1.17 / 1.23, p=0.554 / 0.499)$. However, in patients with $\mathrm{N} 2-3$ or stage IIIB/IIIC disease, $P$ significantly improved survival as compared with S-1. Conversely, S-1 was comparable to $P$ and superior to OBS in stage IB-IIIA or N0-1 disease. S-1 was well-tolerated with a relative dose intensity of $73.6 \%$, a median duration of 8.3 months and associated with less adverse reactions as compared with P. In the subgroup analysis, P was favored in stage III, N2-3 or T3-4 disease wherase patients with Helicobacter Pylori infection had a better survival when treated with S-1.

Conclusions: Adjuvant S-1 correlated with comparable survival rates but fewer adverse reactions as compared with platinum-based doublets. Patients with low to moderate risk of recurrence and Helicobacter pylori infection derived benefits from S-1 while platinum-based doublets were favored in advanced cases. The study provides additional information about adjuvant S-1 in patients with selected risk of recurrence.

\section{Background}

Among all primary gastrointestinal malignancies globally, gastric cancer is highly prevalent and ranks as the 3rd most deadly cancer.[1] The incidence of gastric cancer varies with distinctive geographic and ethnic distributions, where countries in Eastern/Central Asia, Latin America, and Eastern Europe are commonly affected.[2] Despite emerging improvements in early detection and curative treatment, nearly two thirds of high-risk patients still encounter a recurrence or progression of the disease.[3] Therefore, adjuvant or perioperative chemotherapies have been intensively investigated in recent years to reduce distant metastasis. [4-6] Interestingly, the Eastern and Western world share different perspectives of the same disease, in that the former tends to have patients diagnosed earlier, have more lymph nodes dissected, have biologically favorable tumors, and receive more adjuvant chemotherapies and fewer concurrent chemoradiotherapies (CCRT).[7] However, given aggressive salvage therapies, patients with recurrent or advanced gastric cancer still have a dismal outcome with survival barely exceeding more than one year.[8]

$\mathrm{S}-1$, or TS-1, is an orally available chemotherapeutic composed of tegafur (a prodrug of fluorouracil, 5-FU), gimeracil (preventing dihydropyrimidine dehydrogenase-mediated degradation of 5-FU), and oteracil (reducing the toxic effects of 5-FU).[9] It is well-tolerated without serious adverse reactions, easily implemented in an outpatient setting, and currently approved for gastric, lung, breast, and biliary tract cancers in several 
European and Asian countries but not in the United States.[10] S-1 is a key drug replacing the 5-FU backbone and has been incorporated with various chemotherapeutic partners in the treatment of advanced or metastatic gastric cancer in Japanese patients.[11-13] In addition, S-1-based adjuvant therapies, either as mono- or combinatorial regimens, have been confirmed to have efficacy in patients with gastric cancer who have received curative gastrectomy and adequate lymph node dissection (D2 or above) in phase III studies. $[14,15]$ Ongoing studies are investigating neoadjuvant or perioperative chemotherapies containing S-1 as promising options for patients with borderline resectable, locally advanced, or high-risk gastric cancer.[16]

Although S-1 has extended the therapeutic options in adjuvant therapy for gastric cancer, most of the published studies have incorporated patients from Northeast Asia. The extrapolation of the results to other Asian or non-Asian countries remains undetermined. Conversely, genetic and ethnic polymorphisms contribute to differences in drug metabolism, interactions, and dose-limiting toxicities. Pharmacokinetic studies revealed a significantly lower tolerable dose of S-1 in Caucasian patients.[17] In addition, real-world experiences outside of clinical trial settings have been less reported, in which the efficacy of S-1 is potentially confounded by compliance, availability, and undesirable adverse events. In the absence of a universal consensus related to the effective use of adjuvant chemotherapies for resectable gastric cancer, conventional regimens vary across countries. In general, platinum-based doublets, such as cisplatin or oxaliplatin plus a fluoropyrimidine, are widely recommended in the international guidelines.[18] Therefore, a comparison of the efficacy and tolerability of S-1 monotherapy with other adjuvant chemotherapies for patients with resectable gastric cancer warrants further studies, notably real-world experiences in daily clinical practice.

In the present study, we retrospectively evaluated patients with resectable gastric cancer who had received adjuvant S-1 monotherapy post D2 gastrectomy. Patients who received surgery alone or adjuvant platinumbased doublet chemotherapies were enrolled for the purpose of detailed comparisons. Characteristics and survival outcomes were assessed. Also, we reviewed the adverse events and associated factors for S-1 other than platinum-based regimens as selected by physicians. Owing to the differences in baseline disease severity and the nature of retrospective observations, propensity score matching was conducted to enhance between-group comparability. A landscape of recent S-1-based adjuvant or perioperative therapies for gastric cancer was reviewed as well.

\section{Materials And Methods}

\section{Patients}

We included 269 eligible Han Chinese patients with resectable gastric (cardia, fundus, body, antrum, and pylorus) or gastroesophageal junction (GEJ) (Siewert type III tumor) cancer from Jan 2013 to Oct 2018. All patients had an initial American Joint Committee on Cancer (AJCC) pathological staging of IB to III or a restaging of IB to III disease post neoadjuvant therapies. Stage IB patients were excluded if they had T1 disease only or had no high-risk features for recurrence, such as poor differentiation of the tumor, lymphovascular or perineural invasions.[19] Patients were treated and evaluated at National Cheng Kung University Hospital and its affiliated branches in Taiwan, with an average volume of radical gastrectomy of 90 cases per year. Eligible patients were required to have received radical gastrectomy (laparotomic, laparoscopic or robotic) plus perigastric and celiac axial lymph node dissection (D2) in R0 resection without residual tumors. Adjuvant 
chemotherapies were defined as oral or intravenous administration of any single or combinatorial chemotherapeutics within 12 weeks post-operatively for at least two cycles or for 6 consecutive weeks. Eligible chemotherapeutics included S-1 (TTY Biopharm, Taiwan), 5-FU, leucovorin, cisplatin, oxaliplatin, and capecitabine, which were selected based on the physician's clinical judgment. Other additional antineoplastic adjuvant therapies, such as immune checkpoint inhibitors or targeted therapeutics, did not preclude patients from enrollment. Patients were allocated according to the chosen adjuvant chemotherapies as S-1 (Group S1) or platinum-based doublets (Group $P$ ). Those who received close observation with active surveillance without adjuvant therapies were deemed as surgery alone (observation, Group OBS). Patients were excluded if they had carcinoma in situ, initial metastatic or recurrent diseases, adenocarcinoma of unspecified primary sites, secondary cancer with gastric invasion, gastric or GEJ squamous cell carcinoma, small cell carcinoma, lymphoma, or neuroendocrine carcinoma.

\section{Clinical evaluations}

Clinical and pathological features, including demographic characteristics, biochemical markers, Helicobacter pylori (H. pylori) infection, human epidermal growth factor receptor-2 (HER2) status, and treatment outcomes were evaluated based on written or electronic medical records. Chemotherapy-related adverse events were retrospectively retrieved from documented materials and graded according to the Common Toxicity Criteria of the National Cancer Institute (CTCAE) version 4.0. Routine or on-demand esophagogastroduodenoscopy, imaging studies (computed tomography or magnetic resonance imaging), and biopsied or cytological examinations were arranged according to the local practice guidelines and incorporated as the detection of disease recurrence or progression. Recurrence-free survival (RFS) was defined by the date of diagnosis to first documented recurrence or progression of the disease, physician-initiated subsequent therapy, or the death of the patient due to any cause. Overall survival (OS) was defined by the date of diagnosis to the death of the patient due to any cause.

\section{Statistical analysis}

We presented the clinical and pathological features in descriptive analyses as percentages. Continuous variables were compared using a Student's t-test or a one-way analysis of variance (ANOVA) plus a post-hoc Tuckey honestly significant difference (Tuckey's HSD) test, whereas categorical variables were compared using a Chi-squared test or Fisher's exact test.

The variables that did not meet the parametric assumptions were evaluated using non-parametric methods. Propensity scores (PPS) were calculated using a multivariate logistic regression adjusted for the following covariates: age, sex, performance status, AJCC stage, lymph node (N) stage, histology classification, lymphovascular invasion (LVI), and serum CEA level. We conducted PPS matching using the nearest neighbor method at a ratio of $1: 1$ with a caliper of 0.25 to reduce selection bias. If the standardized mean differences are less than $10 \%$, the covariates are considered balanced between the two groups. In addition, we calculated RFS and OS using a Kaplan-Meier survival estimation and compared it with the results of the log-rank test. Uni- and multivariate binary logistic regression analyses were conducted to elucidate independent associated 
factors related to selecting S-1-based adjuvant chemotherapy. Statistical significance was prespecified by $p<0.05$. We used GraphPad Prism 7.0® (GraphPad software, CA, US), SAS 9.4® (SAS Institute Inc., NC, US) and $R \circledast$ 3.5.1 for data management and graphics.

\section{Results}

\section{Patient enrollment and baseline characteristics}

The flow diagram of the study is shown in Fig. 1. A total of 465 patients were screened for enrollment from Jan 2013 to Oct 2018. We excluded 126 patients who were not eligible for the study. Another 70 patients were excluded due to the absence of high-risk features, inadequate lymph node dissection, and adjuvant CCRT. Finally, 269 eligible patients were included in the study. The patient and tumor characteristics are shown in Table 1. Among the included patients, 110 received curative gastrectomy without adjuvant therapies (Group OBS); 64 had S-1 (Group S-1), and 95 had platinum-based doublet (Group P) adjuvant chemotherapies. The distributions of sex, H. pylori infection, HER2 status, baseline performance status, GEJ tumors, and serum cancer antigen 19 - 9 (CA19-9) levels were similar among the three groups. The median ages were similar in patients who received adjuvant chemotherapies, but patients who received surgery alone were significantly older (S-1 vs. P vs. OBS, 65.2 vs. 60.3 vs. 73.3 years). More patients had early stages of the disease, notably stage IB/II, T1-2 or N0 disease, in the Group OBS as compared with Group S- 1 or P (OBS: stage IB/II, $58.2 \%$; T1-2 58.2\%; N0, 53.6\%; all $p<0.001$ when compared with S-1 or P). Similarly, Group S-1 had more stage IB/II and N0 patients as compared with Group P (S-1 vs. P: stage IB/II, 48.4\% vs. $23.2 \%, p=0.002 ; \mathrm{N} 0,21.9 \%$ vs. $4.2 \%, p=0.001$ ). Lymphovascular invasion (LVI) was more evident in Group $\mathrm{S}-1$ and $\mathrm{P}$ as compared with OBS, and the former two had no significant between-group differences (OBS vs. S-1 vs. P: $39.1 \%$ vs. $65.6 \%$ vs. $77.9 \% ; p<0.001$ when OBS was compared with $\mathrm{S} 1$ or $\mathrm{P} ; p=0.088$ in $\mathrm{S} 1$ vs. $\mathrm{P}$ ). Lauren histology classification of the tumors were generally balanced, with the exception of more diffuse type tumors in Group P as compared with OBS or S1 (OBS vs. S-1 vs. P: $30.9 \%$ vs. $43.8 \%$ vs. $51.6 \% ; p=0.009$ in OBS vs. P). The median serum CEA was higher in Group P as compared with S-1 or OBS (OBS vs. S-1 vs. P: 2.1 vs. 1.8 vs. $2.8 \mathrm{ng} / \mathrm{mL}$; $p=0.007$ via a Kruskal-Wallis test). A total of 8 patients in the study had received neoadjuvant therapies prior to surgery, where 3 had triplet; 1 had doublet; 2 had immune checkpoint inhibitors and chemotherapies, and 1 had CCRT. 
Table 1

Patient characteristics

\begin{tabular}{|c|c|c|c|c|c|c|c|c|c|}
\hline \multirow{4}{*}{$\begin{array}{l}\text { Group } \\
\text { Age, median (IQR) }\end{array}$} & \multirow{3}{*}{\multicolumn{2}{|c|}{$\begin{array}{l}\text { OBS } \\
\begin{array}{l}\text { Operation } \\
\text { alone } \\
(n=110)\end{array}\end{array}$}} & \multirow{3}{*}{\multicolumn{2}{|c|}{$\begin{array}{l}S-1 \\
S-1 \\
(n=64)\end{array}$}} & \multirow{3}{*}{\multicolumn{2}{|c|}{$\begin{array}{l}\text { P } \\
\begin{array}{l}\text { Platinum-based } \\
\text { doublets } \\
(\mathrm{n}=95)\end{array}\end{array}$}} & & & \\
\hline & & & & & & & \multicolumn{3}{|l|}{$p$} \\
\hline & & & & & & & \multirow{2}{*}{$\begin{array}{l}\text { OBS } \\
\text { vs. S-1 } \\
< \\
0.001^{*}\end{array}$} & \multirow{2}{*}{$\begin{array}{l}\text { OBS } \\
\text { vs. P } \\
< \\
0.001 *\end{array}$} & \multirow{2}{*}{$\begin{array}{l}\text { S-1 vs. } \\
\text { P } \\
0.066\end{array}$} \\
\hline & 73.3 & $\begin{array}{l}(63.2- \\
81.2)\end{array}$ & 65.2 & $\begin{array}{l}(52.0- \\
70.7)\end{array}$ & 60.3 & $\begin{array}{l}(52.5- \\
68.0)\end{array}$ & & & \\
\hline Male, n (\%) & 63 & (57.3) & 32 & $(50.0)$ & 58 & $(61.1)$ & 0.353 & 0.264 & 0.224 \\
\hline AJCC stage, n (\%) & & & & & & & 0.321 & $\hat{0}_{0.001 *}$ & $0.002 *$ \\
\hline $\mathrm{IB} / \mathrm{II}$ & 64 & $(58.2)$ & 31 & $(48.4)$ & 22 & $(23.2)$ & & & \\
\hline IIIA & 14 & & 13 & & 14 & & & & \\
\hline IIIB/IIIC & 32 & & 20 & & 59 & & & & \\
\hline \multicolumn{10}{|l|}{ pT stage, n (\%) } \\
\hline $\mathrm{T} 1 / 2$ & 64 & $(58.2)$ & 13 & $(20.3)$ & 18 & $(18.9)$ & $\hat{0}_{0.001 *}$ & $\hat{0}_{0.001 *}$ & 0.992 \\
\hline T3 & 34 & & 32 & & 38 & & & & \\
\hline $\mathrm{T} 4$ & 12 & & 19 & & 39 & & & & \\
\hline pN stage, $n(\%)$ & & & & & & & $<0.001 *$ & $<.001 *$ & $0.001 *$ \\
\hline NO & 59 & $(53.6)$ & 14 & $(21.9)$ & 4 & $(4.2)$ & & & \\
\hline $\mathrm{N} 1 / 2$ & 42 & & 36 & & 40 & & & & \\
\hline N3 & 9 & & 14 & & 51 & & & & \\
\hline$E C O G \geq 2, n(\%)$ & 13 & $(11.8)$ & 4 & (6.3) & 8 & (8.4) & 0.233 & 0.424 & 0.840 \\
\hline $\begin{array}{l}\text { HER2-positive, n } \\
(\%)\end{array}$ & 15 & $(13.6)$ & 4 & (6.3) & 9 & (9.5) & 0.132 & 0.355 & 0.665 \\
\hline HER2-therapy ${ }^{\mathrm{a}}, \mathrm{n}$ & & 0 & & 2 & & & & & \\
\hline $\begin{array}{l}\text { Tumor at GEJ, } n \\
(\%)\end{array}$ & 7 & (6.3) & 3 & $(4.7)$ & 9 & (9.5) & 0.647 & 0.408 & 0.415 \\
\hline Lauren histology, $\mathrm{n}$ & & & & & & & 0.195 & $0.009 *$ & 0.600 \\
\hline Intestinal & 57 & $(51.8)$ & 25 & $(39.1)$ & 32 & $(33.7)$ & & & \\
\hline Diffuse & 34 & $(30.9)$ & 28 & $(43.8)$ & 49 & $(51.6)$ & & & \\
\hline Mixed & 16 & & 10 & & 14 & & & & \\
\hline
\end{tabular}




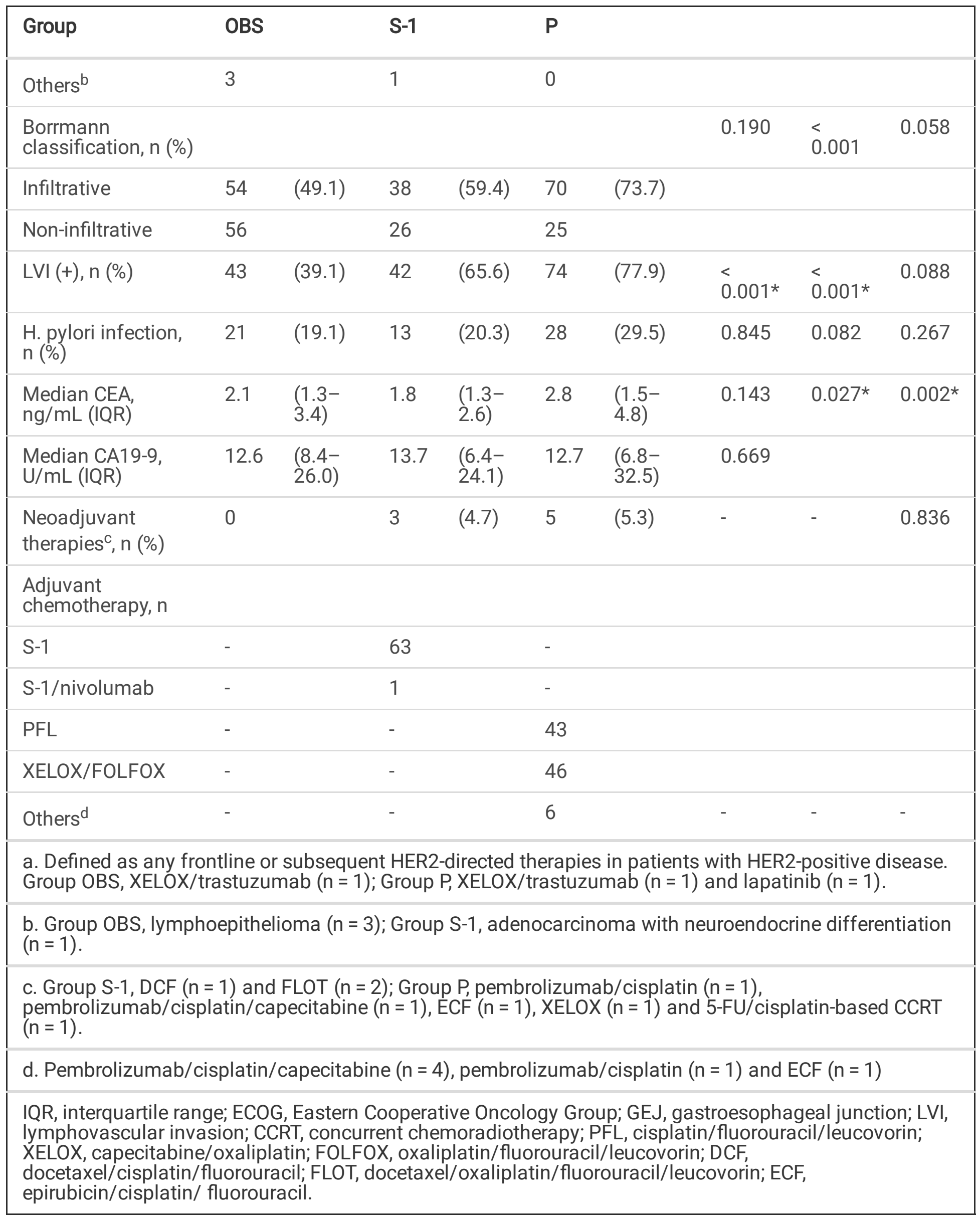




\section{PPS matching and post-match characteristics}

Two independent matched results are demonstrated in Table 2. A total of 61 and 58 patients were matched in the analysis 1 (S-1 vs. P) and 2 (S-1 vs. OBS), respectively. Both post-match analyses revealed well-balanced characteristics in terms of age, sex, performance status, stage (disease, T, or N stage), and histology classification, with the exception of marginal disproportions in stage IB/II disease in analysis 1 and serum CEA in analysis 2. In Group S-1, all patients had S-1 monotherapy as adjuvant chemotherapy. In Group P, 29/61 patients had cisplatin/5-FU/leucovorin (PFL); 30/61 patients had oxaliplatin-based doublets, and 2/61 patients had pembrolizumab/cisplatin/capecitabine as the selected adjuvant chemotherapies. 
Table 2

Post matching characteristics

\begin{tabular}{|c|c|c|c|c|c|c|c|c|c|c|}
\hline \multirow{4}{*}{ Group } & \multicolumn{5}{|c|}{ Analysis $1(1: 1)$} & \multicolumn{5}{|c|}{ Analysis $2(1: 1)$} \\
\hline & \multicolumn{2}{|l|}{$S-1$} & \multicolumn{3}{|c|}{ P-based } & \multicolumn{2}{|l|}{$S-1$} & \multicolumn{3}{|l|}{ OBS } \\
\hline & \multirow{2}{*}{\multicolumn{2}{|c|}{$\begin{array}{l}S-1 \\
(n=61)\end{array}$}} & \multirow{2}{*}{\multicolumn{2}{|c|}{$\begin{array}{l}\text { Platinum- } \\
\text { based }(n=61)\end{array}$}} & \multirow[t]{2}{*}{$p$} & \multirow{2}{*}{\multicolumn{2}{|c|}{$\begin{array}{l}S-1 \\
(n=58)\end{array}$}} & \multirow{2}{*}{\multicolumn{2}{|c|}{$\begin{array}{l}\text { Surgery alone } \\
(n=58)\end{array}$}} & \multirow[t]{2}{*}{$p$} \\
\hline & & & & & & & & & & \\
\hline $\begin{array}{l}\text { Age, median } \\
\text { (IQR) }\end{array}$ & 65.0 & $\begin{array}{l}(55.9- \\
75.3)\end{array}$ & 61.8 & $\begin{array}{l}(55.5- \\
69.4)\end{array}$ & 0.544 & 65.5 & $\begin{array}{l}(57.8- \\
76.0)\end{array}$ & 70.1 & $\begin{array}{l}(60.6- \\
76.2)\end{array}$ & 0.143 \\
\hline Male, n (\%) & 31 & $(51.7)$ & 33 & $(54.1)$ & 0.784 & 29 & $(50.0)$ & 31 & $(53.4)$ & 0.999 \\
\hline $\begin{array}{l}\text { Disease stage, } \\
\mathrm{n}(\%)\end{array}$ & & & & & 0.470 & & & & & 0.370 \\
\hline $\mathrm{IB} / \mathrm{II}$ & 25 & $(40.1)$ & 19 & (31.1) & & 31 & $(53.4)$ & 33 & $(56.9)$ & \\
\hline IIIA & 13 & & 13 & & & 9 & & 13 & & \\
\hline IIIB/IIIC & 23 & & 29 & & & 18 & & 12 & & \\
\hline pT stage, n (\%) & & & & & 0.825 & & & & & 0.999 \\
\hline $\mathrm{T} 1 / 2$ & 12 & (19.7) & 14 & $(23.0)$ & & 13 & $(22.4)$ & 13 & $(22.4)$ & \\
\hline T3/4 & 49 & & 47 & & & 45 & & 45 & & \\
\hline pN stage, n (\%) & & & & & 0.499 & & & & & 0.980 \\
\hline No & 11 & $(18.0)$ & 7 & $(11.5)$ & & 14 & $(24.1)$ & 16 & $(27.6)$ & \\
\hline $\mathrm{N} 1 / 2$ & 36 & & 36 & & & 32 & & 31 & & \\
\hline N3 & 14 & & 18 & & & 12 & & 11 & & \\
\hline$E C O G \geq 2, n(\%)$ & 4 & $(6.6)$ & 5 & $(8.2)$ & 0.729 & 4 & $(6.9)$ & 8 & $(13.8)$ & 0.222 \\
\hline $\begin{array}{l}\text { HER2-positive, n } \\
(\%)\end{array}$ & 4 & $(6.6)$ & 5 & (8.2) & 0.729 & 3 & $(5.2)$ & 6 & $(10.3)$ & 0.298 \\
\hline $\begin{array}{l}\text { Tumor at GEJ, } n \\
(\%)\end{array}$ & 2 & (3.3) & 3 & $(4.9)$ & 0.995 & 3 & $(5.2)$ & 3 & $(5.2)$ & 0.999 \\
\hline $\begin{array}{l}\text { Lauren } \\
\text { histology type, } \\
\text { n (\%) }\end{array}$ & & & & & 0.509 & & & & & 0.287 \\
\hline Intestinal & 24 & (39.3) & 23 & (37.7) & & 23 & $(39.7)$ & 28 & $(48.3)$ & \\
\hline Diffuse & 26 & $(42.6)$ & 31 & $(50.8)$ & & 25 & $(43.1)$ & 19 & $(32.8)$ & \\
\hline Mixed & 10 & & 7 & & & 9 & & 10 & & \\
\hline \multicolumn{11}{|c|}{ a. Adenocarcinoma with neuroendocrine differentiation $(n=1)$} \\
\hline b. Pembrolizuma & /cispla & /capec & abine ( & $=2)$ & & & & & & \\
\hline
\end{tabular}




\begin{tabular}{|c|c|c|c|c|c|c|c|c|c|c|}
\hline \multirow[b]{2}{*}{ Others ${ }^{a}$} & \multicolumn{5}{|c|}{ Analysis $1(1: 1)$} & \multicolumn{5}{|c|}{ Analysis $2(1: 1)$} \\
\hline & 1 & & 0 & & & 1 & & 0 & & \\
\hline $\begin{array}{l}\text { Borrmann } \\
\text { classification, n } \\
(\%)\end{array}$ & & & & & 0.253 & & & & & 0.699 \\
\hline Infiltrative & 37 & $(60.7)$ & 43 & $(70.5)$ & & 38 & $(65.5)$ & 36 & $(62.1)$ & \\
\hline Non-infiltrative & 24 & & 18 & & & 20 & & 22 & & \\
\hline LVI (+), n (\%) & 42 & $(68.9)$ & 49 & $(80.3)$ & 0.145 & 31 & $(53.4)$ & 36 & $(62.1)$ & 0.347 \\
\hline $\begin{array}{l}\text { Median CEA } \\
\text { ng/mL, (IQR) }\end{array}$ & 1.9 & $\begin{array}{l}(1.2- \\
2.7)^{-}\end{array}$ & 1.9 & $\begin{array}{l}\left(1.2^{-}\right. \\
3.1)^{-}\end{array}$ & 0.535 & 1.9 & $\begin{array}{l}(1.3- \\
2.6)\end{array}$ & 2.1 & $\begin{array}{l}(1.5- \\
4.4)\end{array}$ & 0.072 \\
\hline $\begin{array}{l}\text { H. pylori } \\
\text { infection, n (\%) }\end{array}$ & 12 & (19.7) & 16 & $(26.2)$ & 0.389 & 13 & $(22.4)$ & 10 & $(17.2)$ & 0.485 \\
\hline \multicolumn{11}{|l|}{$\begin{array}{l}\text { Adjuvant } \\
\text { chemotherapy, } \\
\text { n (\%) }\end{array}$} \\
\hline S-1 & 61 & & - & & & 58 & & - & & \\
\hline PFL & - & & 29 & & & - & & - & & \\
\hline XELOX/FOLFOX & - & & 30 & & & - & & - & & \\
\hline Others $^{b}$ & - & & 2 & & & - & & - & & \\
\hline \multicolumn{11}{|c|}{ a. Adenocarcinoma with neuroendocrine differentiation $(n=1)$} \\
\hline b. Pembrolizuma & /cisp & in/capec & abine & $=2)$ & & & & & & \\
\hline
\end{tabular}

\section{Survival: S-1 vs. platinum-based doublets}

All patients were evaluated in a median follow-up time of 42.5 months. In analysis 1 , patients who received adjuvant S-1 had a trend of shorter RFS and OS as compared with platinum-based doublets but it did not reach statistical significance (median RFS/OS: S-1 vs. P, 24.4/37.4 vs. 39.1/62.1 months, $\mathrm{HR}=1.12 / 1.22, p=$ $0.667 / 0.514$; Fig. $2 \mathrm{~A}$ and $2 \mathrm{~B}$ ). Stratified by the $\mathrm{N}$ stage, the patients in Group P had significantly better RFS and OS in N2-3 disease as compared with Group S-1 (median RFS/OS: S-1 vs. P, 19.3/27.8 vs. 28.5/56.0 months, $\mathrm{HR}=2.04 / 2.29, p=0.010 / 0.007$; Fig. $2 \mathrm{C}$ and $2 \mathrm{D}$ ). However, the survival differences were not evident in terms of N0-1 disease (5-year RFS/OS rates: S-1 vs. P, 66.5\%/67.1\% vs. 60.0\%/71.0\%, HR= 0.65/0.57, $p=$ $0.458 / 0.479$; Fig. $2 E$ and $2 F$ ). Stratified by staging, patients with stage IB to IIIA disease had comparable RFS and OS (5-year RFS/OS rates: S-1 vs. P, $62.3 \% / 66.3 \%$ vs. 52.8\%/71.3\%, HR=0.73/0.60, $p=0.431 / 0.371$; Supplementary $1 \mathrm{~A}$ and $1 \mathrm{~B})$. However, platinum-based doublets were associated with significantly prolonged survival rates in patients with stage IIIB/IIIC disease (median RFS/OS: S-1 vs. P, 12.8/27.0 vs. 28.5/55.6 months, $\mathrm{HR}=2.87 / 2.89, p=0.001 / 0.002$; Supplementary $1 \mathrm{C}$ and $1 \mathrm{D}$ ). We did not observe significant survival differences based on the S-1, oxaliplatin, or cisplatin-based chemotherapy in the matched population (median 
RFS: S-1 vs. oxaliplatin vs. cisplatin, 24.4 vs. 24.7 vs. 54.3 months; all $p \geq 0.05$ between groups;

Supplementary 1E) (median OS: S-1 vs. oxaliplatin vs. cisplatin, 37.4 vs. 55.67 vs. 64.2 months; all $p \geq 0.05$ between groups; Supplementary $1 \mathrm{~F})$.

\section{Survival: S-1 vs. OBS}

In analysis 2, Group S-1 had a comparable survival outcome to that of OBS (median RFS/OS: S-1 vs. OBS, 23.6/37.4 vs. 24.0/64.6 months, $\mathrm{HR}=1.17 / 1.23, p=0.554 / 0.499 ;$ Fig. 3A and 3B). Conversely, patients with N0-1 disease in Group S-1 had a significantly better RFS and OS (median RFS/OS: S-1 vs. OBS, undefined/37.4 vs. undefined/61.0 months, $\mathrm{HR}=0.41 / 0.27, p=0.038 / 0.024 ;$ Fig. $3 \mathrm{C}$ and 3D), but the survival advantage disappeared in N2-3 disease as compared with OBS (median RFS/OS: S-1 vs. OBS, 16.1/27.8 vs. 16.2/28.9 months, HR=1.10/1.03, $p=0.778 / 0.948$; Fig. 3E and 3F). Stratified by staging, stage IB to IIIA patients in Group S-1 had a longer RFS and OS (5-yr RFS/OS rates: S-1 vs. OBS, 71.0\%/65.4\% vs. $50.1 \% / 56.9 \%, H R=0.41 / 1.03, p=0.041 / 0.032$; Supplementary 2A and 2B). However, dismal survival outcomes were observed in the stage IIIB and IIIC patients, irrespective of whether they received adjuvant S-1 or not (median RFS/OS: S-1 vs. OBS, 12.8/21.0 vs. 11.8/25.9 months, HR=0.95/1.09, $p=0.871 / 0.841$; Supplementary 2C and 2D).

\section{Adverse events and dosage intensity}

We present the adverse events and the grading in Table 3 for the total number of evaluable patients (S-1 monotherapy, $n=61$; platinum-based doublets, $n=84$ ). There were no deaths attributable to the adjuvant chemotherapies. Anemia was the most common adverse event in all patients and was significantly higher in the platinum-based doublets as compared with S-1 (grade III/IV anemia: S-1 vs. combination therapy, $4.9 \%$ vs. $9.5 \%, p=0.028$ ). Grade III/IV thrombocytopenia, renal insufficiency, and neutropenia were prevalent in the platinum-based doublets (thrombocytopenia, 17.9\%; renal insufficiency, 4.8\%; neutropenia, 3.6\%). Scattered cases of severe diarrhea, anorexia, mucositis, and palmar plantar erythrodysesthesia were also observed in patients who received platinum-based doublets. In the S-1 group, except for a few cases with grade III/IV hepatitis $(n=2)$, thrombocytopenia $(n=1)$, and neutropenia $(n=1)$, there were no additional warning toxicities reported. One patient developed S-1-related grade III drug eruptions that were relieved after discontinuation and supportive care. The average dose intensity of S-1 was $40.9 \pm 13.6 \mathrm{mg}$ in patients with a body surface area $(B S A)<1.25 \mathrm{~m}^{2}, 47.4 \pm 13.4 \mathrm{mg}$ with a BSA from 1.25 to $1.50 \mathrm{~m}^{2}$, and $58.5 \pm 12.4 \mathrm{mg}$ with a BSA $>1.50$ $\mathrm{m}^{2}$ per day, which were lower than the suggested dose from the published studies, with an average relative intensity of $73.6 \%$ (Supplement 3 ). $10.9 \%$ of the patients required a dose reduction due to intolerable toxicity. The median duration of S-1 was 8.3 months, respectively. 
Table 3

Adverse events, according to the treatment

\begin{tabular}{|c|c|c|c|c|c|c|c|c|c|}
\hline \multirow{2}{*}{ Events } & \multicolumn{4}{|c|}{$\begin{array}{l}S-1 \\
(n=61)\end{array}$} & \multicolumn{5}{|c|}{$\begin{array}{l}\text { Platinum-based doublets } \\
(\mathrm{n}=84)^{\mathrm{a}}\end{array}$} \\
\hline & $\begin{array}{l}\text { Total, } \\
\mathrm{n}\end{array}$ & $\begin{array}{l}\text { Grade } \\
\mathrm{I} / \mathrm{II}, \mathrm{n}\end{array}$ & $\begin{array}{l}\text { Grade } \\
\text { III/IV, } \\
\mathrm{n}\end{array}$ & $\begin{array}{l}\text { Grade } \\
\text { III/IV, } \\
(\%)\end{array}$ & $\begin{array}{l}\text { Total, } \\
\text { n }\end{array}$ & $\begin{array}{l}\text { Grade } \\
\mathrm{I} / \mathrm{Il}, \mathrm{n}\end{array}$ & $\begin{array}{l}\text { Grade } \\
\text { III/IV } \\
\mathrm{n}\end{array}$ & $\begin{array}{l}\text { Grade } \\
\text { III/IV, } \\
(\%)\end{array}$ & $\mathrm{p}^{\mathrm{b}}$ \\
\hline Any events & 90 & 82 & 8 & - & 212 & 173 & 39 & - & - \\
\hline Anemia & 22 & 19 & 3 & 4.9 & 47 & 39 & 8 & 9.5 & $0.028^{*}$ \\
\hline Thrombocytopenia & 7 & 6 & 1 & 1.6 & 36 & 21 & 15 & 17.9 & <.001* \\
\hline Neutropenia & 3 & 2 & 1 & 1.6 & 12 & 9 & 3 & 3.6 & 0.121 \\
\hline Renal insufficiency & 5 & 5 & 0 & 0 & 17 & 13 & 4 & 4.8 & $0.046^{\star}$ \\
\hline Hepatitis & 13 & 11 & 2 & 3.3 & 15 & 13 & 2 & 2.3 & 0.759 \\
\hline Anorexia & 22 & 22 & 0 & 0 & 37 & 33 & 4 & 4.8 & 0.427 \\
\hline Diarrhea & 9 & 9 & 0 & 0 & 8 & 7 & 1 & 1.2 & 0.481 \\
\hline Vomiting & 6 & 6 & 0 & 0 & 29 & 29 & 0 & 0 & $0.001 *$ \\
\hline Mucositis & 1 & 1 & 0 & 0 & 4 & 3 & 1 & 1.2 & 0.398 \\
\hline PPE & 0 & 0 & 0 & 0 & 6 & 5 & 1 & 1.2 & - \\
\hline Skin eruptions & 2 & 1 & 1 & 1.6 & 1 & 0 & 1 & 1.2 & 0.573 \\
\hline \multicolumn{10}{|c|}{ a. XELOX $(n=41), \operatorname{PFL}(n=38)$ and FOLFOX $(n=5)$. } \\
\hline \multicolumn{10}{|c|}{ b. Comparisons with incidence of all events. } \\
\hline \multicolumn{10}{|c|}{ PPE, palmar plantar erythrodysesthesia } \\
\hline
\end{tabular}

\section{Subgroup analysis}

The subgroup analyses for RFS and OS are shown in Fig. 4 using forest plotting. In the total population, we did not observe significant differences among those treated with adjuvant S-1 or platinum-based doublets. In the subgroups, patients with stage III, T3-4, or N2-3 disease had a longer RFS on platinum-based doublets. Female gender, stage III or N2-3 disease were associated with favorable OS when treated with platinum-based doublets. To the contrary, H. pylori infection correlated with a survival benefit in patients who received S-1.

\section{Factors associated with adjuvant S-1}


We evaluated the factors associated with selecting adjuvant S-1 versus platinum-based doublets using a binary logistic regression, and the results are shown in Table 4. We observed that low serum CEA, N0-2 disease, increased patient age, low serum CA19-9, and early stages were associated factors in the univariate regression. Following multivariate adjustments, low serum CEA, NO-2 disease, and increased patient age were significant factors for adjuvant S-1 rather than platinum-based doublets. 
Table 4

Associated factors for the selection of adjuvant S-1

\begin{tabular}{|c|c|c|c|c|c|c|c|}
\hline & \multicolumn{4}{|c|}{ Univariate regression } & \multicolumn{3}{|c|}{ Multivariate regression } \\
\hline & $\mathrm{n}$ & $\mathrm{OR}$ & $95 \% \mathrm{Cl}$ & $\mathbf{p}$ & OR & $95 \% \mathrm{Cl}$ & p \\
\hline CEA & 40 & 1.00 & $2.11-13.79$ & $<0.001 *$ & 3.93 & $1.44-10.7$ & $0.008^{*}$ \\
\hline$\geq 5 \mathrm{ng} / \mathrm{mL}$ & 119 & 5.39 & & & & & \\
\hline \multicolumn{8}{|l|}{$<5 \mathrm{ng} / \mathrm{mL}$} \\
\hline $\mathrm{N}$ stage & 65 & 1.00 & $1.60-7.09$ & $0.001^{*}$ & 2.16 & $1.02-5.01$ & $0.044^{*}$ \\
\hline N3 & 75 & 3.36 & $3.13-33.20$ & $<0.001^{*}$ & 5.48 & $1.21-24.90$ & $0.028 *$ \\
\hline N1-2 & 19 & 10.2 & & & & & \\
\hline \multicolumn{8}{|l|}{ NO } \\
\hline Age, increased by 1 year & 159 & 1.03 & $0.99-1.05$ & 0.058 & 1.04 & $1.01-1.07$ & $0.041 *$ \\
\hline CA199 & 23 & 1.00 & $1.21-11.61$ & $0.022^{*}$ & 2.82 & $0.77-10.42$ & 0.118 \\
\hline$\geq 47 \mathrm{IU} / \mathrm{mL}$ & 136 & 3.75 & & & & & \\
\hline \multicolumn{8}{|l|}{$<47 \mathrm{IU} / \mathrm{mL}$} \\
\hline AJCC stage & 107 & 1.00 & $1.66-6.60$ & $0.001^{*}$ & 1.74 & $0.69-4.41$ & 0.241 \\
\hline III & 52 & 3.31 & & & & & \\
\hline \multicolumn{8}{|l|}{ I/II } \\
\hline Gender & 90 & 1.00 & $0.83-2.97$ & 0.169 & & & \\
\hline Male & 69 & 1.57 & & & & & \\
\hline \multicolumn{8}{|l|}{ Female } \\
\hline H. pylori infection & 41 & 1.00 & $0.77-3.48$ & 0.197 & & & \\
\hline Presence & 118 & 1.64 & & & & & \\
\hline \multicolumn{8}{|l|}{ Absence } \\
\hline LVI & 118 & 1.00 & $0.78-3.28$ & 0.198 & & & \\
\hline Presence & 41 & 1.60 & & & & & \\
\hline Absence & & & & & & & \\
\hline
\end{tabular}

OR, odds ratio 


\begin{tabular}{|c|c|c|c|c|c|c|c|}
\hline & \multicolumn{4}{|c|}{ Univariate regression } & \multicolumn{3}{|c|}{ Multivariate regression } \\
\hline & $\mathbf{n}$ & OR & $95 \% \mathrm{Cl}$ & $\mathrm{p}$ & OR & $95 \% \mathrm{Cl}$ & p \\
\hline Lauren classification & 55 & 1.00 & $0.36-1.47$ & 0.381 & & & \\
\hline Intestinal & 77 & 0.73 & $0.39-2.59$ & 0.991 & & & \\
\hline Diffuse & 27 & 1.01 & & & & & \\
\hline \multicolumn{8}{|l|}{ Mixed } \\
\hline T stage & 31 & 1.17 & $0.50-2.74$ & 0.725 & & & \\
\hline T1-2 & 70 & 0.68 & $0.27-1.66$ & 0.391 & & & \\
\hline T3 & 58 & & & & & & \\
\hline \multicolumn{8}{|l|}{$\mathrm{T} 4$} \\
\hline Tumor differentiation & 44 & 1.00 & $0.37-1.50$ & 0.409 & & & \\
\hline Well/Moderate & 115 & 0.74 & & & & & \\
\hline \multicolumn{8}{|l|}{ Poor } \\
\hline ECOG & 147 & 1.00 & $0.40-4.79$ & 0.613 & & & \\
\hline$<2$ & 12 & 1.38 & & & & & \\
\hline \multicolumn{8}{|l|}{$\geq 2$} \\
\hline HER2 status & 13 & 1.00 & $0.43-2.85$ & 0.829 & & & \\
\hline Positive & 146 & 1.11 & & & & & \\
\hline \multicolumn{8}{|l|}{ Negative } \\
\hline Borrmann classification & 41 & 1.00 & $0.52-2.22$ & 0.852 & & & \\
\hline Non-infiltrative & 118 & 1.07 & & & & & \\
\hline Infiltrative & & & & & & & \\
\hline OR, odds ratio & & & & & & & \\
\hline
\end{tabular}

\section{Discussion}

Adjuvant S-1 has extended the therapeutic options in stage II and III gastric cancer patients who have received D2 gastrectomy, notably in Asia.[18] The present study provides additional information on S-1 versus conventional platinum-based chemotherapies based on real-world practice. Our results indicated that patients with early stages of the disease had comparable outcomes on adjuvant S-1 as compared with platinumbased doublets. Conversely, S-1 monotherapy was associated with fewer adverse reactions and better tolerability. In an era prompting aggressive adjuvant therapies for high-risk diseases, curative gastrectomy alone is no longer being considered as a standard of care. Sakuramoto et al. proposed that adjuvant S-1 is 
superior to D2 gastrectomy alone in stage II and III gastric cancer in terms of both recurrence-free and overall survival. $[14,20]$ In the CLASSIC study, adjuvant capecitabine/oxaliplatin (XELOX) significantly improved survival outcomes as compared with surgery only at the cost of more adverse events.[21] In patients with a particularly high risk of recurrence, the role of more aggressive therapies has been intensively investigated. The ARTIST-2 trial evaluated the role of adjuvant S-1/oxaliplatin (SOX), SOX plus CCRT (SOXRT), and S-1 monotherapy in lymph node-positive patients, where it was concluded that S-1-based doublets, irrespective of whether the patient underwent radiotherapy or not, were superior to S-1 in terms of disease-free survival and met the prespecified endpoint early.[22] Yoshida et al. also reported the therapeutic efficacy of docetaxel/S-1 in stage III, $\geq T 2$ or lymph node-positive patients versus S-1, with a significantly higher incidence of grade III to IV treatment-related toxicities.[15] Other combinations, such as oxaliplatin or cisplatin plus S-1, have been considered to be feasible options but were found to be relatively more toxic than S-1 monotherapy.[11, 23-25] Our results suggest that adjuvant S-1 is well-tolerated and comparable in efficacy to other combinatorial regimens in patients with moderate to low risk of recurrence. Although the results of intensive protocols are promising, selecting an optimal adjuvant chemotherapy remains a difficult task that requires a balance between the therapeutic benefits and toxicity.

Despite the fact that we did not discover a significant difference between adjuvant S-1 versus platinum-based doublets in the total population, subgroups of patients still shared distinctive outcomes. Patients with $H$. pylori infection had more favorable survival when treated with S-1. H. pylori played an unclear role as a prognostic factor in patients with gastric cancer.[26, 27] However, Nishizuka et al. reported that $H$. pyloriinfected patients had superior survival rates when treated with adjuvant $\mathrm{S}-1$, which was compatible with our results. [28] Host antitumor immunity may be enhanced by $H$. pylori infection and fluoropyrimidine-mediated eradication of myeloid-derived suppressor cells, which may contribute to a survival advantage.[29] Furthermore, we noticed that the survival outcomes in stage IB-IIIA or N0-1 disease were better with S-1, whereas platinum-based doublets led to additional benefits in the more advanced diseases. In the ACTS-GC study, the therapeutic efficacy of S-1 was less evident in patients with stage III, T3-4 disease, or more than 3 involved lymph nodes.[20] A retrospective analysis indicated that patients with stage IIIC, N3 disease, or a lymph node ratio $(L N R)>0.25$ did better with adjuvant XELOX rather than with S-1.[30] Hsieh et al. also suggested that stage III or LNR $>0.21$ are significant prognostic factors in patients receiving S-1 versus non-S1 doublet chemotherapies.[31] Similarly, in the JACCRO-GC-07 trial, despite the fact that docetaxel/S-1 was superior to S-1 monotherapy in the primary analysis, the benefit disappeared in patients with N0-1 disease. [15] Our results, in line with the published reports, indicated a definitive role of adjuvant S-1 in patients with selected risk of recurrence post curative surgery.

Good tolerance and limited warning toxicities with adjuvant S-1 was observed in the present study. We found only a few cases of grade III to IV events, such as anemia, hepatitis, and skin eruptions in contrast to a higher risk of anemia, thrombocytopenia, neutropenia, and renal insufficiency with the use of platinum-based doublets. In addition, a low demand of dose reduction (10.9\%) was noticed, despite the fact that the patients were potentially under-dosed $(73.6 \%$ as compared with reference trials) in a real-world practice environment. Kim et al. reported a dose of S-1 at $40 \mathrm{mg} / \mathrm{m}^{2}$ twice daily in D1-28 every 6 weeks for one year and concluded that $46.3 \%$ of the patients required a dose modification, and $16.1 \%$ discontinued treatment due to persistent toxicity.[32] Yamatsuji et al. compared a 4-week administration period followed by two weeks of rest (4-w/2-

Page $17 / 27$ 
w) with a two week administration period followed by one week of rest (2-w/1-w) and revealed that the latter had a higher completion rate, higher relative dose intensity, and similar toxicities.[33] In addition, the optimal dosage of S-1 is potentially confounded by ethnicity. Yang et al. revealed that S-1 at a dose of $35 \mathrm{mg} / \mathrm{m}^{2}$ twice daily was better tolerated in Asian patients in combination with oxaliplatin.[34] However, another pharmacokinetic study revealed a poor tolerance in a Western population, and the dose was reduced to 25

$\mathrm{mg} / \mathrm{m}^{2}$ twice daily.[17] The discrepancy is partially derived from the pharmacogenetic polymorphisms of cytochrome P 2A6 (CYP2A6), which contribute to a distinctive S-1 elimination efficiency.[35] Further studies are required to determine the patient-tailored optimal dosage of $\mathrm{S}-1$, both in mono- or combinatorial therapies.

We found that physicians preferred S-1 as adjuvant chemotherapy in patients with low CEA, N0-2 disease, and increased age. Lee et al. suggested that S-1 is more likely to be selected as adjuvant chemotherapy for patients over 65 years of age, whereas XELOX was selected for patients with stage III disease, corresponding to our results.[36] Terashima et al. evaluated the tumor gene expression profiles of 102 patients who received adjuvant chemotherapies via a DNA microarray and found that the cluster of differentially expressed genes that favored S-1 was enriched with immune-related genes.[37] Together, these results highlight physician preferences and potential selection biomarkers for those who may derive benefits from S-1 containing adjuvant therapies. Recently, perioperative chemotherapies have gained awareness for the efficacy on locallyadvanced or high-risk patients with resectable or borderline resectable gastric cancer. Various combinations of fluoropyrimidines, platinums, anthracyclines, or taxanes have been adopted to improve the resectability, surgical outcome, and long-term survival, as well as to reduce recurrence.[4-6, 21, 38, 39] The addition of S-1 to these agents is being intensively investigated.[40-42] Furthermore, the results are promising in the combination of S-1, nivolumab, oxaliplatin, and capecitabine in advanced or recurrent gastric cancer.[43] More clinical studies are required to delineate the role of $\mathrm{S}-1$, both in the frontline or adjuvant setting and in the treatment of gastric cancer.

The strength of the present study is the real-world information of S-1 outside of clinical trial settings, which is more closely related to actual clinical scenarios. In addition, we incorporated PPS matching to exclude baseline heterogeneity and enhance between-group comparability. Third, we provided the dosages and adverse reactions for S-1 in comparison with other combinatorial regimens. However, there are some limitations to this study. It is a retrospective analysis that cannot address prospective consequential imputations. Also, we included patients who received S-1 monotherapy in the absence of S-1-based combinations, which have gradually become the standard of care in high-risk populations. In addition, despite the efforts we made to control for differences of patients via PPS matching, we still were unable to eradicate possible exclusion bias and undetermined imbalances. Lastly, the patients were collected from a single institution, and the case number was not significantly large enough to have an excellent statistical power. Overall, the study still provided information concerning adjuvant S-1 in the treatment of resectable gastric cancer and a comparison with close observation and platinum-based doublets.

\section{Conclusion}

In the present study, patients with D2-resected stage IB-III gastric cancer who received adjuvant S-1 had comparable survival rates and fewer adverse reactions as compared with those receiving platinum-based

Page $18 / 27$ 
doublets. Patients with low to moderate risk of recurrence and $H$. pylori infection derived more benefits from S-1, while platinum-based doublets were found to be superior in advanced diseases. S-1 was relatively underdosed and was found to be selected by physicians based on serum CEA, lymph node status, and patient age. More prospective studies are required to delineate the optimal selection of patients who may benefit from S-1 or S-1-based combinations in gastric cancer.

\section{List Of Abbreviations}

S-1, tegafur/gimeracil/octeracil; OBS, observation; RFS, recurrence-free; OS, overall survivals; CCRT, concurrent chemoradiotherapies; GEJ, gastroesophageal junction; AJCC, American Joint Committee on Cancer; H. pylori, Helicobacter pylori; CTCAE, Common Toxicity Criteria of the National Cancer Institute; ANOVA, analysis of variance; PPS, propensity scores; CA19-9, cancer antigen 19-9; LVI, lymphovascular invasion; PFL, cisplatin/5FU/leucovorin; LNR, lymph node ratio; CYP, cytochrome P.

\section{Declarations}

\section{Ethics approval and consent to participate}

This is a retrospective observational study including patients with standard medical care. All analyzed information had received a valid de-identification process. Therefore, the informed consent is waived as approved by the Institutional Review Board (IRB) at National Cheng Kung University Hospital, Tainan, Taiwan. The study is approved by the registry serial number B-ER-109-184 in accordance with the Declaration of Helsinki and its amendments.

\section{Consent for publication}

Not applicable

\section{Availability of data and materials}

The data that support the findings of this study are available from Cancer Registry of the Electronic Medical Records in National Cheng Kung University Hospital but restrictions apply to the availability of these data, which were used under specific permission for the current study, and so are not publicly available. Data are however available from the authors upon reasonable request and with permission of Clinical Research Center, National Cheng Kung University Hospital, Tainan, Taiwan. em74820@mail.hosp.ncku.edu.tw. No. 138, ShengLi Road, Tainan 70403, Taiwan. Tel: +886-6-235-3535 ext. 4817.

\section{Competing interests}

The authors have no conflicts of interest to declare. 


\section{Funding}

The study was funded by Clinical Research Center, National Cheng Kung University Hospital and has no other funding sources elsewhere.

\section{Authors' contributions}

Chih-Chieh Yen is the first author of the study and was responsible for writing the manuscript and data management. Yan-Shen Shan is the second author, who provided critical advice and suggestions for revisions. Ting-Kai Liao, Yin-Jui Chao, Hsuan-Yi Huang and I-Ting Liu are the third group of authors, who contributed to the writing of the manuscript. Chia-Jui Yen is the corresponding author who initiated the study design and investigations.

\section{Acknowledgement}

The authors thank the staff and research nurses at National Cheng Kung University Hospital Cancer Center for recording the clinical information and medical records.

\section{References}

1. Rawla P, Barsouk A: Epidemiology of gastric cancer: global trends, risk factors and prevention. Prz Gastroenterol 2019, 14(1):26-38.

2. Balakrishnan M, George R, Sharma A, Graham DY: Changing Trends in Stomach Cancer Throughout the World. Curr Gastroenterol Rep 2017, 19(8):017-0575.

3. Shin C-H, Lee $W-Y$, Hong S-W, Chang Y-G: Characteristics of gastric cancer recurrence five or more years after curative gastrectomy. Chin J Cancer Res 2016, 28(5):503-510.

4. Cunningham D, Allum WH, Stenning SP, Thompson JN, Van de Velde CJ, Nicolson M, Scarffe JH, Lofts FJ, Falk SJ, Iveson TJ et al: Perioperative chemotherapy versus surgery alone for resectable gastroesophageal cancer. N Engl J Med 2006, 355(1):11-20.

5. Ychou M, Boige V, Pignon JP, Conroy T, Bouché O, Lebreton G, Ducourtieux M, Bedenne L, Fabre JM, SaintAubert $\mathrm{B}$ et al: Perioperative chemotherapy compared with surgery alone for resectable gastroesophageal adenocarcinoma: an FNCLCC and FFCD multicenter phase III trial. J Clin Oncol 2011, 29(13):1715-1721.

6. Al-Batran SE, Homann N, Pauligk C, Goetze TO, Meiler J, Kasper S, Kopp HG, Mayer F, Haag GM, Luley K et al: Perioperative chemotherapy with fluorouracil plus leucovorin, oxaliplatin, and docetaxel versus fluorouracil or capecitabine plus cisplatin and epirubicin for locally advanced, resectable gastric or gastro-oesophageal junction adenocarcinoma (FLOT4): a randomised, phase 2/3 trial. Lancet 2019, 393(10184):1948-1957.

7. Bickenbach K, Strong VE: Comparisons of Gastric Cancer Treatments: East vs. West. J Gastric Cancer 2012, 12(2):55-62. 
8. Wagner AD, Unverzagt S, Grothe W, Kleber G, Grothey A, Haerting J, Fleig WE: Chemotherapy for advanced gastric cancer. Cochrane Database Syst Rev 2010, 17(3).

9. Shirasaka T, Shimamato Y, Ohshimo H, Yamaguchi M, Kato T, Yonekura K, Fukushima M: Development of a novel form of an oral 5-fluorouracil derivative (S-1) directed to the potentiation of the tumor selective cytotoxicity of 5-fluorouracil by two biochemical modulators. Anticancer Drugs 1996, 7(5):548-557.

10. Chhetri P, Giri A, Shakya S, Shakya S, Sapkota B, Pramod KC: Current Development of Anti-Cancer Drug S1. J Clin Diagn Res 2016, 10(11):XE01-XE05.

11. Koizumi W, Narahara H, Hara T, Takagane A, Akiya T, Takagi M, Miyashita K, Nishizaki T, Kobayashi O, Takiyama W et al: S-1 plus cisplatin versus S-1 alone for first-line treatment of advanced gastric cancer (SPIRITS trial): a phase III trial. Lancet Oncol 2008, 9(3):215-221.

12. Tsuburaya A, Sakamoto J, Morita S, Kodera Y, Kobayashi M, Miyashita Y, Macdonald JS: A randomized phase III trial of post-operative adjuvant oral fluoropyrimidine versus sequential paclitaxel/oral fluoropyrimidine; and UFT versus S1 for T3/T4 gastric carcinoma: the Stomach Cancer Adjuvant Multiinstitutional Trial Group (Samit) Trial. Jpn J Clin Oncol 2005, 35(11):672-675.

13. Yamada Y, Higuchi K, Nishikawa K, Gotoh M, Fuse N, Sugimoto N, Nishina T, Amagai K, Chin K, Niwa Y et al: Phase III study comparing oxaliplatin plus S-1 with cisplatin plus S-1 in chemotherapy-na\&\#xef;ve patients with advanced gastric cancer. Annals of Oncology 2015, 26(1):141-148.

14. Sakuramoto S, Sasako M, Yamaguchi T, Kinoshita T, Fujii M, Nashimoto A, Furukawa H, Nakajima T, Ohashi $\mathrm{Y}$, Imamura $\mathrm{H}$ et al: Adjuvant chemotherapy for gastric cancer with $\mathrm{S}-1$, an oral fluoropyrimidine. $N$ Engl J Med 2007, 357(18):1810-1820.

15. Yoshida K, Kodera Y, Kochi M, Ichikawa W, Kakeji Y, Sano T, Nagao N, Takahashi M, Takagane A, Watanabe $T$ et al: Addition of Docetaxel to Oral Fluoropyrimidine Improves Efficacy in Patients With Stage III Gastric Cancer: Interim Analysis of JACCRO GC-07, a Randomized Controlled Trial. Journal of clinical oncology : official journal of the American Society of Clinical Oncology 2019, 37(15):1296-1304.

16. Iwasaki Y, Terashima M, Mizusawa J, Katayama H, Nakamura K, Katai H, Yoshikawa T, Ito Y, Kaji M, Kimura $Y$ et al: Randomized phase III trial of gastrectomy with or without neoadjuvant S-1 plus cisplatin for type 4 or large type 3 gastric cancer: Japan Clinical Oncology Group study (JCOG0501). Journal of Clinical Oncology 2018, 36(15_suppl):4046-4046.

17. Ajani JA, Faust J, Ikeda K, Yao JC, Anbe H, Carr KL, Houghton M, Urrea P: Phase I Pharmacokinetic Study of S-1 Plus Cisplatin in Patients With Advanced Gastric Carcinoma. Journal of Clinical Oncology 2005, 23(28):6957-6965.

18. Kim I-H: Current status of adjuvant chemotherapy for gastric cancer. World J Gastrointest Oncol 2019, 11(9):679-685.

19. Smyth EC, Verheij M, Allum W, Cunningham D, Cervantes A, Arnold D: Gastric cancer: ESMO Clinical Practice Guidelines for diagnosis, treatment and follow-up. Ann Oncol 2016, 27(suppl 5):v38-v49.

20. Sasako M, Sakuramoto S, Katai H, Kinoshita T, Furukawa H, Yamaguchi T, Nashimoto A, Fujii M, Nakajima T, Ohashi Y: Five-year outcomes of a randomized phase III trial comparing adjuvant chemotherapy with S-1 versus surgery alone in stage II or III gastric cancer. J Clin Oncol2011, 29(33):4387-4393. 
21. Bang Y-J, Kim Y-W, Yang H-K, Chung HC, Park Y-K, Lee KH, Lee K-W, Kim YH, Noh S-I, Cho JY et al: Adjuvant capecitabine and oxaliplatin for gastric cancer after D2 gastrectomy (CLASSIC): a phase 3 open-label, randomised controlled trial. Lancet 2012, 379(9813):315-321.

22. Park SH, Zang DY, Han B, Ji JH, Kim TG, Oh SY, Hwang IG, Kim JH, Shin D, Lim DH et al: ARTIST 2: Interim results of a phase III trial involving adjuvant chemotherapy and/or chemoradiotherapy after D2gastrectomy in stage II/III gastric cancer (GC). Journal of Clinical Oncology 2019, 37(15_suppl):40014001.

23. Lee C-k, Jung M, Kim HS, Jung I, Shin DB, Kang SY, Zang DY, Kim KH, Lee MH, Kim B-S et al: S-1 Based Doublet as an Adjuvant Chemotherapy for Curatively Resected Stage III Gastric Cancer: Results from the Randomized Phase III POST Trial. Cancer Res Treat 2019, 51(1):1-11.

24. Yamada Y, Higuchi K, Nishikawa K, Gotoh M, Fuse N, Sugimoto N, Nishina T, Amagai K, Chin K, Niwa Y et al: Phase III study comparing oxaliplatin plus S-1 with cisplatin plus S-1 in chemotherapy-naive patients with advanced gastric cancer. Ann Oncol 2015, 26(1):141-148.

25. Shitara K, Chin K, Yoshikawa T, Katai H, Terashima M, Ito S, Hirao M, Yoshida K, Oki E, Sasako M et al: Phase II study of adjuvant chemotherapy of S-1 plus oxaliplatin for patients with stage III gastric cancer after D2 gastrectomy. Gastric Cancer 2017, 20(1):175-181.

26. Meimarakis G, Winter H, Assmann I, Kopp R, Lehn N, Kist M, Stolte M, Jauch KW, Hatz RA: Helicobacter pylori as a prognostic indicator after curative resection of gastric carcinoma: a prospective study. Lancet Oncol 2006, 7(3):211-222.

27. Li G, Wang Z, Xu J, Cui J, Cai S, Zhan W, He Y: Gastric cancer patients with Helicobacter pylori infection have a poor prognosis. J Surg Oncol 2013, 108(7):421-426.

28. Nishizuka SS, Tamura G, Nakatochi M, Fukushima N, Ohmori Y, Sumida C, Iwaya T, Takahashi T, Koeda K: Helicobacter pylori infection is associated with favorable outcome in advanced gastric cancer patients treated with S-1 adjuvant chemotherapy. J Surg Oncol 2018, 117(5):947-956.

29. Vincent J, Mignot G, Chalmin F, Ladoire S, Bruchard M, Chevriaux A, Martin F, Apetoh L, Rébé C, Ghiringhelli F: 5-Fluorouracil Selectively Kills Tumor-Associated Myeloid-Derived Suppressor Cells Resulting in Enhanced T Cell-Dependent Antitumor Immunity. Cancer Research 2010, 70(8):3052.

30. Shin K, Park SJ, Lee J, Park CH, Song KY, Lee HH, Seo HS, Jung YJ, Park JM, Lee SH et al: Efficacy of capecitabine and oxaliplatin versus S-1 as adjuvant chemotherapy in gastric cancer after D2 lymph node dissection according to lymph node ratio and N stage. BMC Cancer 2019, 19(1):1232.

31. Hsieh MC, Wang SH, Wei CT, Chen CY, Chen YY, Pei SN, Tsai YF, Rau KM: S1 versus Doublet Regimens as Adjuvant Chemotherapy in Patients with Advanced Gastric Cancer after Radical Surgery with D2 Dissection-A Propensity Score Matching Analysis. Cancers 2020, 12(9).

32. Kim SJ, Kim YJ, Kim JH, Park DJ, Kim HH, Lee JS, Lee KW: Safety, compliance, and predictive parameters for dosage modification in adjuvant S-1 chemotherapy for gastric cancer. Cancer Sci 2013, 104(1):116123.

33. Yamatsuji T, Fujiwara Y, Matsumoto H, Hato S, Namikawa T, Hanazaki K, Takaoka M, Hayashi J, Shigemitsu K, Yoshida K et al: Feasibility of oral administration of S-1 as adjuvant chemotherapy in gastric cancer: 4-week S-1 administration followed by 2-week rest vs. 2-week administration followed by 1-week rest. Mol Clin Oncol 2015, 3(3):527-532. 
34. Yang L, Yang Y, Qin Q, Zhou A, Zhao J, Wang J, Shu C, Yuan X, Hu S: Evaluation of the optimal dosage of S-1 in adjuvant SOX chemotherapy for gastric cancer. Oncol Lett 2015, 9(3):1451-1457.

35. Chuah B, Goh B-C, Lee S-C, Soong R, Lau F, Mulay M, Dinolfo M, Lim S-E, Soo R, Furuie T et al: Comparison of the pharmacokinetics and pharmacodynamics of S-1 between Caucasian and East Asian patients. Cancer Science 2011, 102(2):478-483.

36. Lee HY, Hwang IG, Park S-E, Kim MJ, Park SH, Kang JH, Kim YS, Oh SY, Won Y-W, Lee SI et al: Factors Influencing Clinicians' Choice of Adjuvant S-1 versus Capecitabine plus Oxaliplatin after Curative Gastrectomy in Patients with Gastric Cancer. J Cancer 2016, 7(12):1711-1715.

37. Terashima M, Nakamura K, Hatakeyama K, Furukawa K, Fujiya K, kamiya S, Hikage M, Tanizawa Y, Bando E, Oshima K et al: Prediction of S-1 adjuvant chemotherapy efficacy in stage II/III gastric cancer treatment based on comprehensive gene expression analysis. Annals of Oncology 2019, 30:v315.

38. Alderson D, Cunningham D, Nankivell M, Blazeby JM, Griffin SM, Crellin A, Grabsch HI, Langer R, Pritchard S, Okines A et al: Neoadjuvant cisplatin and fluorouracil versus epirubicin, cisplatin, and capecitabine followed by resection in patients with oesophageal adenocarcinoma (UK MRC OE05): an open-label, randomised phase 3 trial. The Lancet Oncology 2017, 18(9):1249-1260.

39. Schuhmacher C, Gretschel S, Lordick F, Reichardt P, Hohenberger W, Eisenberger CF, Haag C, Mauer ME, Hasan B, Welch J et al: Neoadjuvant Chemotherapy Compared With Surgery Alone for Locally Advanced Cancer of the Stomach and Cardia: European Organisation for Research and Treatment of Cancer Randomized Trial 40954. Journal of Clinical Oncology 2010, 28(35):5210-5218.

40. Terashima M, Iwasaki Y, Mizusawa J, Katayama H, Nakamura K, Katai H, Yoshikawa T, Ito Y, Kaji M, Kimura $Y$ et al: Randomized phase III trial of gastrectomy with or without neoadjuvant S-1 plus cisplatin for type 4 or large type 3 gastric cancer, the short-term safety and surgical results: Japan Clinical Oncology Group Study (JCOG0501). Gastric Cancer 2019, 22(5):1044-1052.

41. Tokunaga M, Mizusawa J, Machida N, Fukagawa T, Katai H, Nishida Y, Yabusaki H, Ito S, Sano T, Sasako $\mathrm{M}$ et al: Phase III trial to evaluate the efficacy of neoadjuvant chemotherapy with S-1 plus oxaliplatin followed by D2 gastrectomy with adjuvant S-1 in locally advanced gastric cancer: Japan Clinical Oncology Group study JCOG1509 (NAGISA trial). Journal of Clinical Oncology 2017, 35(15_suppl):TPS4134-TPS4134.

42. Wang Y, Cheng X, Cui Y-H, Hou J, Ji Y, Sun Y-H, Shen Z-B, Liu F-L, Liu T-S: Efficacy after preoperative capecitabine and oxaliplatin (XELOX) versus docetaxel, oxaliplatin and S1 (DOS) in patients with locally advanced gastric adenocarcinoma: a propensity score matching analysis. BMC Cancer 2018, 18(1):702702.

43. Boku N, Ryu MH, Kato K, Chung HC, Minashi K, Lee KW, Cho H, Kang WK, Komatsu Y, Tsuda M et al: Safety and efficacy of nivolumab in combination with S-1/capecitabine plus oxaliplatin in patients with previously untreated, unresectable, advanced, or recurrent gastric/gastroesophageal junction cancer: interim results of a randomized, phase II trial (ATTRACTION-4). Annals of Oncology 2019, 30(2):250-258.

\section{Figures}




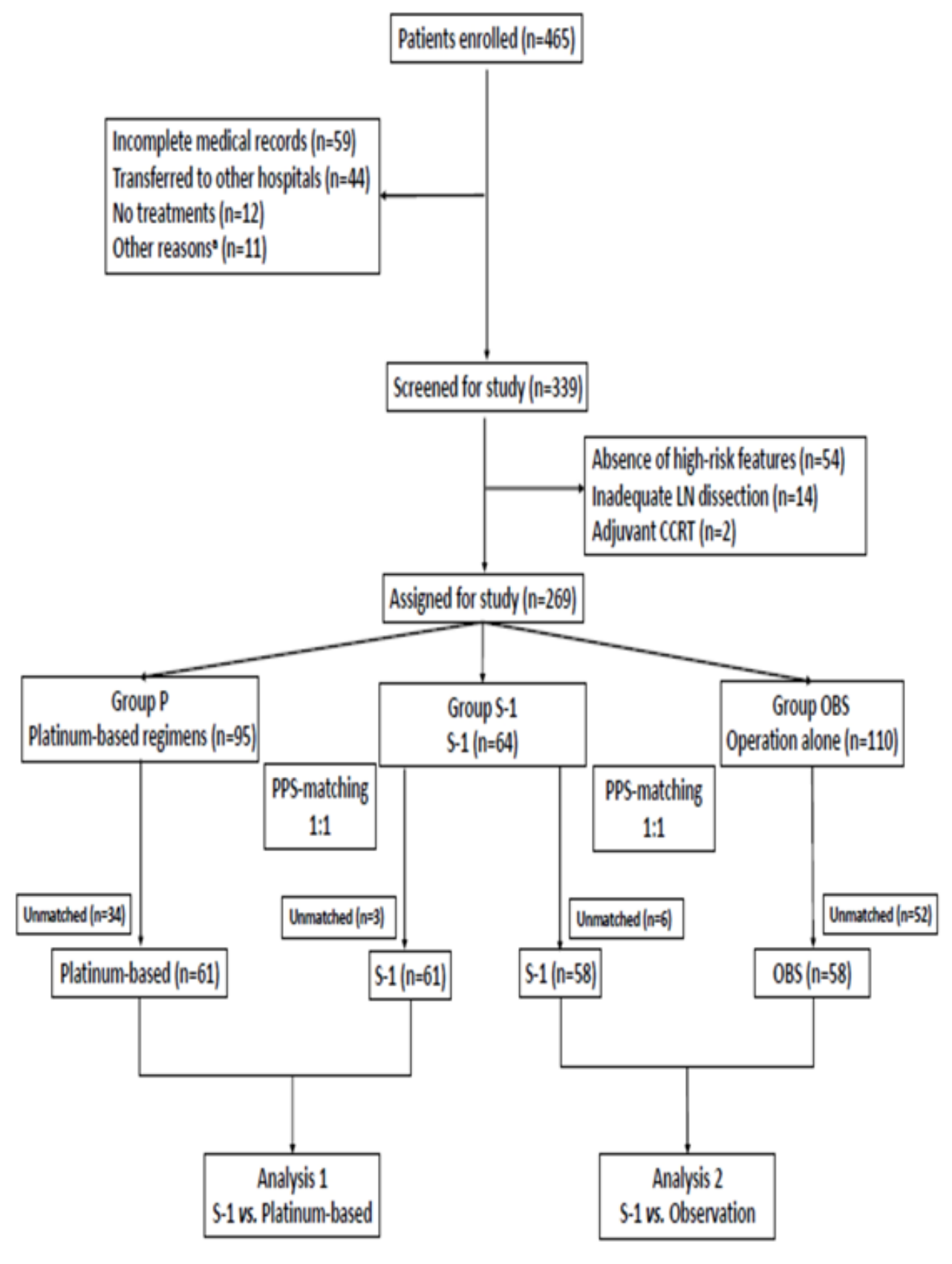

\section{Figure 1}

Flow diagram of the study a. Concomitant malignancies other than gastric cancer $(n=6)$, metastatic carcinoma not confined to gastric origin $(n=3)$, and esophageal carcinoma with gastric invasion $(n=2)$. CCRT, concurrent chemoradiotherapy; LN, lymph node; PPS, propensity score. 

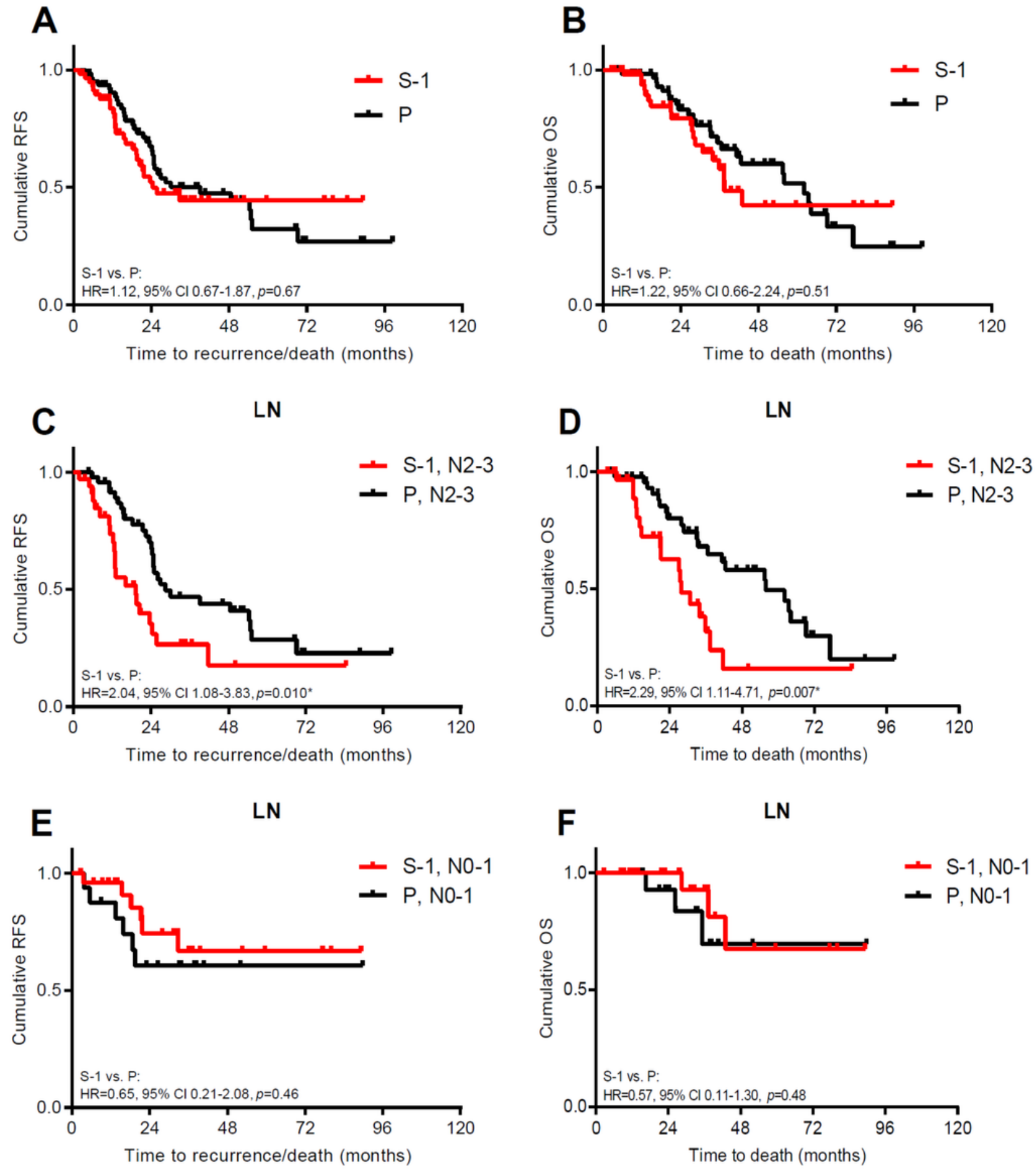

\section{Figure 2}

Survival: S-1 vs. platinum-based doublets RFS and OS by adjuvant chemotherapy (A-B), lymph node stage of N2-3 (C-D), and NO-1 (E-F). LN, lymph node stage; HR, hazard ratio; Cl, confidence interval. Log-rank test, $\mathrm{p}<$ 0.05 as statistically significant and shown as *. 

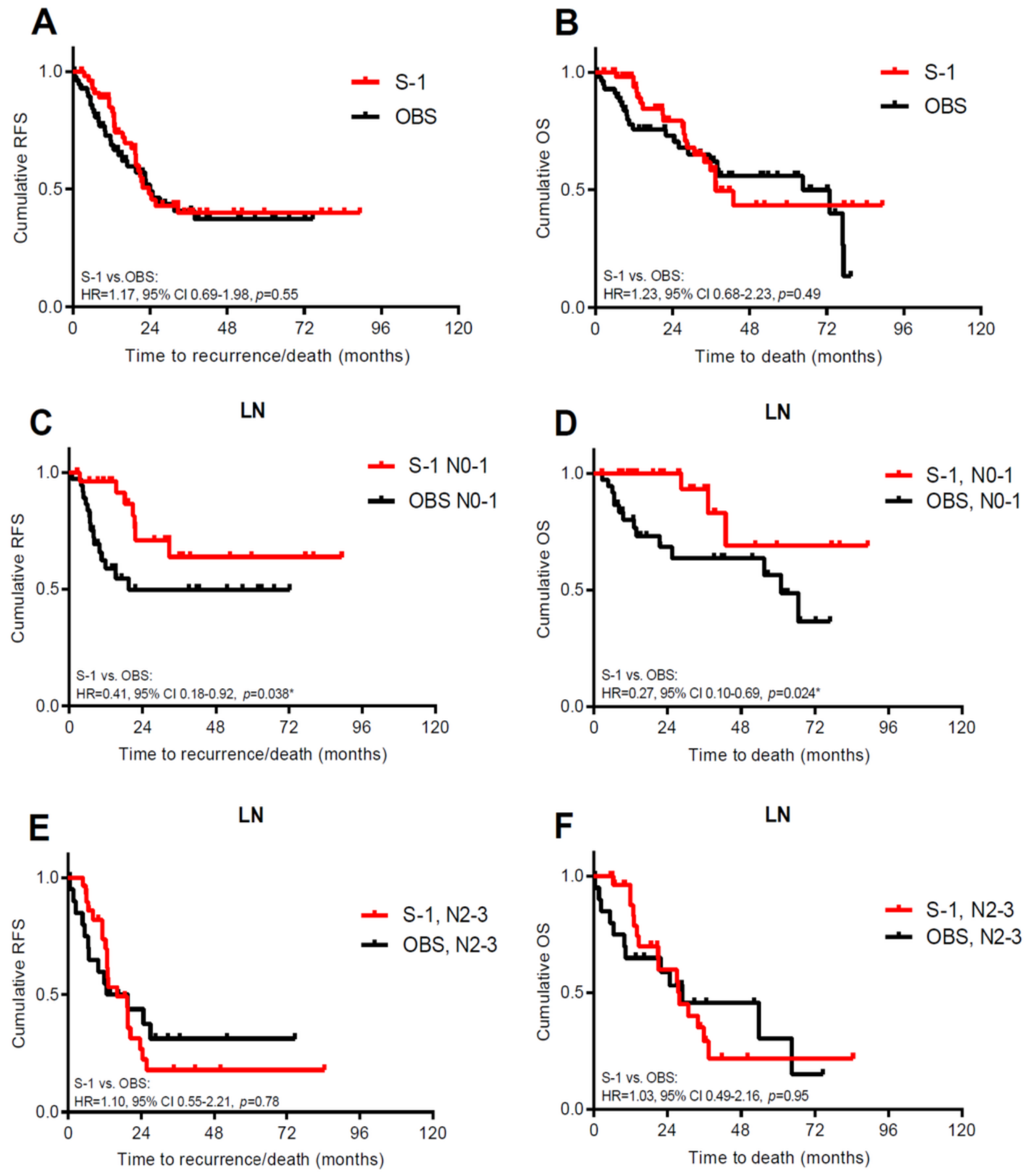

\section{Figure 3}

Survival: S-1 vs. close observation RFS and OS by adjuvant S-1 or OBS (A-B), lymph node stage of N0-1 (C-D), and N2-3 (E-F). Log-rank test, $p<0.05$ as statistically significant and shown as *. 


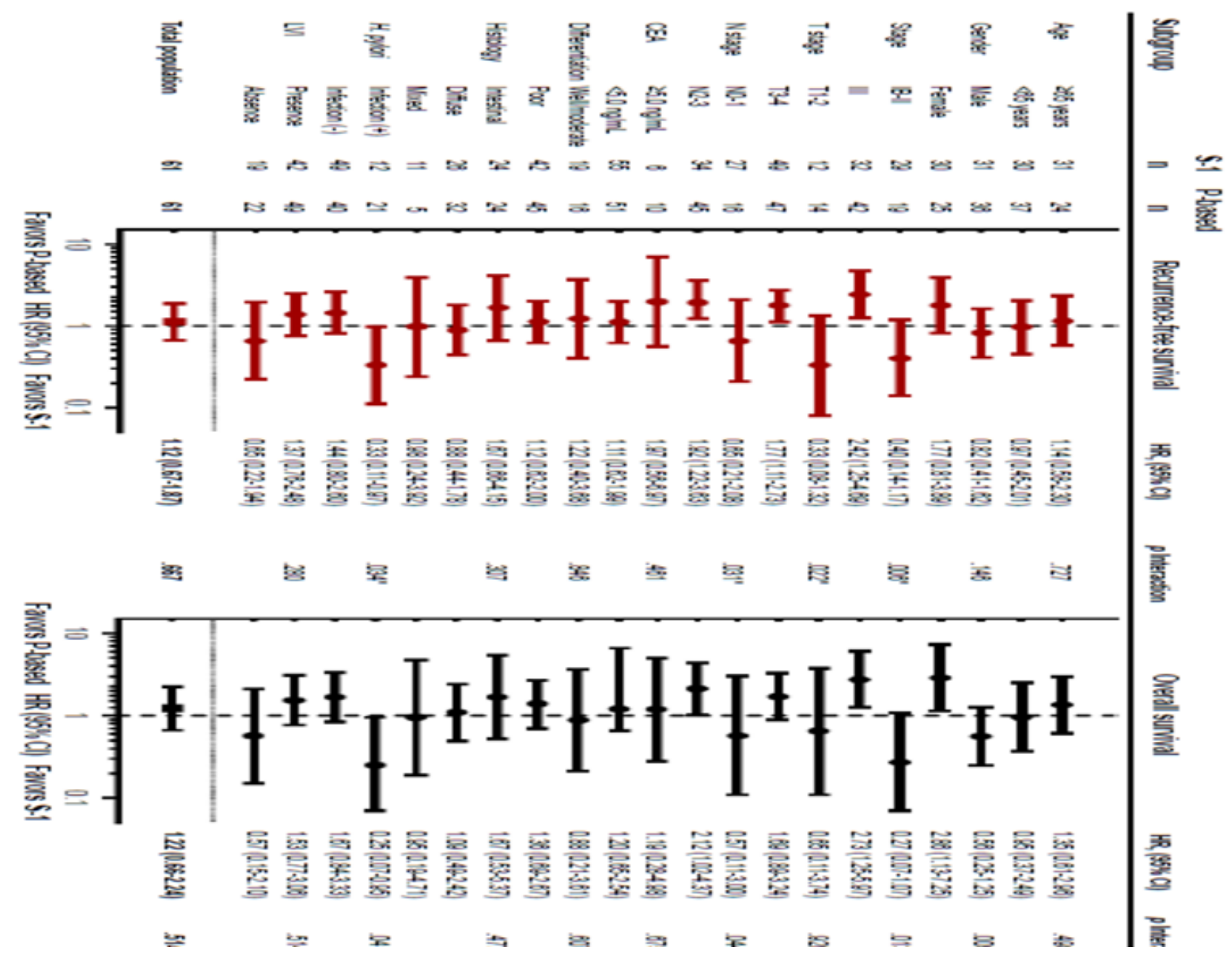

Figure 4

Subgroup analysis $p$ for interaction $<0.05$ as statistically significant and shown as *

\section{Supplementary Files}

This is a list of supplementary files associated with this preprint. Click to download.

- Supplementarymaterials.pdf 